\title{
Análise da estabilidade elástica em torres tubulares de aço para aerogeradores de eixo horizontal
}

\author{
Douglas Mateus de Lima ${ }^{1}$, Pablo Aníbal López-Yánez² e José Weslen da Silva ${ }^{3}$ \\ ${ }^{1}$ Professor do Núcleo de Tecnologia, Universidade Federal de Pernambuco - \\ CAA, Caruaru-PE, douglasortoedro@gmail.com \\ 2 Professor do Departamento de Engenharia Civil, Universidade Federal de \\ Pernambuco - CTG, Recife-PE, lopez.yanez@yahoo.com.br \\ ${ }^{3}$ Graduando em Engenharia Civil na Universidade Federal de Pernambuco - \\ CAA, Caruaru-PE, weslen.eng.civil@gmail.com
}

\section{Elastic stability analysis in steel tubular towers for horizontal axis wind turbines}

\section{Resumo}

Neste trabalho, apresenta-se e discute-se o comportamento da estabilidade do conjunto estrutural formado por uma torre tubular de aço com $120 \mathrm{~m}$ de altura e por sua fundação (sapata). Inicialmente, escreveu-se a equação diferencial ordinária da torre que foi modelada via método das diferenças finitas para obterem-se os seus deslocamentos transversais. Em seguida, o projeto do modelo de torre e da sua fundação foi realizado conforme os principais códigos normativos. Então, a torre, a fundação e a interação solo-estrutura foram modeladas via método dos elementos finitos. Constatou-se que ocorre levantamento da sapata em virtude da flexibilidade do sistema fundação-solo, resultando em um incremento no deslocamento transversal total medido no topo da torre; nesta situação, a estabilidade do conjunto foi confirmada.

Palavras-chave: estabilidade, torres tubulares de aço, aerogeradores, energia eólica.

\begin{abstract}
In this paper, the stability behavior of the structural assembly formed by a $120 \mathrm{~m}$ high steel tower and its foundation (slab) is presented and discussed. Initially, it was written the ordinary differential equation of the tower that was modeled by finite difference method to obtain its transverse displacements. Next, the design of the tower model and its foundation was carried out according to the main normative codes. Then, the tower, the foundation and the soilstructure interaction were modeled via the finite element method. It was found that a slab foundation lifts due to the flexibility of the foundation-soil system, resulting in an increment on the total transverse displacement at the top of the tower; regarding these conditions, global stability was verified.
\end{abstract}

Keywords: stability, steel tubular towers, wind turbines, wind energy.

\footnotetext{
*Autor correspondente
} 


\section{Introdução}

No início do século XXI, teve-se um crescimento acelerado na implantação de aerogeradores, onshore e offshore, de porte crescente com torres cada vez mais altas (Engström et al., 2010). O desenvolvimento, o comércio e a instalação de aerogeradores no mundo se desenvolveram rapidamente, de forma que a geração de energia a partir de termoelétricas, usina nucleares e hidrelétricas tenha sido complementada e/ou substituída pela produção daqueles equipamentos.

A geração de energia elétrica por meio de turbinas eólicas constitui uma alternativa para diversos níveis de demanda no Brasil. As pequenas centrais podem suprir pequenas localidades distantes da rede de distribuição; já às centrais de grande porte têm potencial para atender uma significativa parcela do Sistema Interligado Nacional (SIN) com importantes ganhos. Especificamente no Nordeste brasileiro (especialmente dos estados da Paraíba, Rio Grande do Norte, Ceará, Piauí e Pernambuco), o desenvolvimento da produção de energia eólica se deu de maneira promissora nos últimos anos, pois diversas usinas eólicas estão em operação e em fase de implantação, fazendo com que a geração de energia elétrica de origem eólica tenha crescido exponencialmente na última década (BBC BRASIL, 2015).

Aliado ao exposto acima, a evolução do tamanho dos aerogeradores, cada vez mais pesados e potentes, torna necessária a instalação destes equipamentos sob a ação de ventos mais intensos e contínuos, fazendo com que as dimensões das torres destes aerogeradores estejam sendo incrementadas. Particularmente, a altura da torre é um parâmetro essencial para captação de ventos estáveis de grande altura; entretanto, o custo da torre, que pode superar $20 \%$ do custo total do gerador eólico (Hau, 2006), faz com que o aumento de altura represente uma desvantagem. Além disto, o transporte, a montagem e a posta em operação da torre tornam-se mais custosos.

Adicionalmente, o incremento da esbelteza das torres resulta num aumento dos efeitos de $2^{a}$ ordem a que estas estruturas ficam submetidas e, concomitantemente, agravam a probabilidade de tombamento do conjunto fundação-torre-nacele-rotor. Este fato leva à necessidade de estudos mais detalhados para a previsão de deslocamentos e deformações, tanto da torre quanto fundação. Alguns autores, a exemplo de Bazeos et 
al. (2002) e Lavassas et al. (2003), estudaram questões relacionadas com o projeto e com as análises estruturais estáticas, de estabilidade e de comportamento sísmico, de protótipos de torre com 38 e 45 m de altura para aerogeradores com potências nominais de 0,75 e $1 \mathrm{MW}$, respectivamente. Ademais, Sirqueira (2008) estudou o comportamento estrutural de uma torre com 76,2 m de altura para um aerogerador com $2 \mathrm{MW}$ de potência nominal.

Entretanto, percebeu-se a necessidade de estudos nacionais e regionais a respeito da estabilidade e do projeto de torres tubulares de aço para aerogeradores de maior porte. Portanto, neste artigo, são apresentados o projeto estrutural e a análise de estabilidade de um conjunto de torre tubular de aço, com 120 m de altura, e sua fundação para um aerogerador de grande porte com potência nominal de 3,2 MW. Portanto, o objetivo deste artigo é realizar uma análise detalhada da estabilidade elástica da estrutura composta solo-fundação-torre, de maneira a fornecer subsídios ao desenvolvimento das análises de tais estruturas, uma vez que, por exemplo, alguns tipos de carregamento, como a carga de neve, são considerados no projeto de torres que são projetadas na Europa, mas são fabricadas e utilizadas no nordeste brasileiro, onde tais carregamentos não se aplicam.

\section{Modelo Teórico}

\subsection{Mecânica do meio contínuo (M. M. C.)}

Para a análise estrutural da torre tubular utilizaram-se as equações do equilíbrio da vigacoluna (Figura 1), assim, considerando-se um elemento infinitesimal de torre e analisando-se o equilíbrio de momentos em torno do ponto $A$, obtém-se:

$$
-M-\frac{q(d x)^{2}}{2}+p p d x \frac{d v}{2}-(V+d V) d x+M+d M-(N+d N) d v=0
$$

Na Equação (1): $x$ é a coordenada ao longo da altura da torre; $M=M(x)$ é a função de momento fletor; $V=V(x)$ é a função de esforço transversal; $N=N(x)$ é a função de esforço axial; $q=q(x)$ é a função de carregamento transversal; $p p=p p(x)$ é a função de carregamento axial; e, $\mathrm{v}=\mathrm{v}(\mathrm{x})$ é a função de deslocamento transversal da torre. Simplificando-se a Equação 1, resulta:

$$
V=\frac{d M}{d x}-N \frac{d v}{d x}
$$


e avaliando-se o equilíbrio de forças na direção transversal, tem-se:

$$
V-q d x-(V+d V)=0
$$

em que, simplificando-se, resulta:

$$
q=-\frac{d V}{d x}
$$

Analisando-se agora o equilíbrio de forças na direção axial, tem-se:

$$
N+p p d x-(N+d N)=0
$$

ou, ainda, simplificando-se esta expressão, obtém-se:

$$
\mathrm{pp}=\frac{\mathrm{dN}}{\mathrm{dx}}
$$

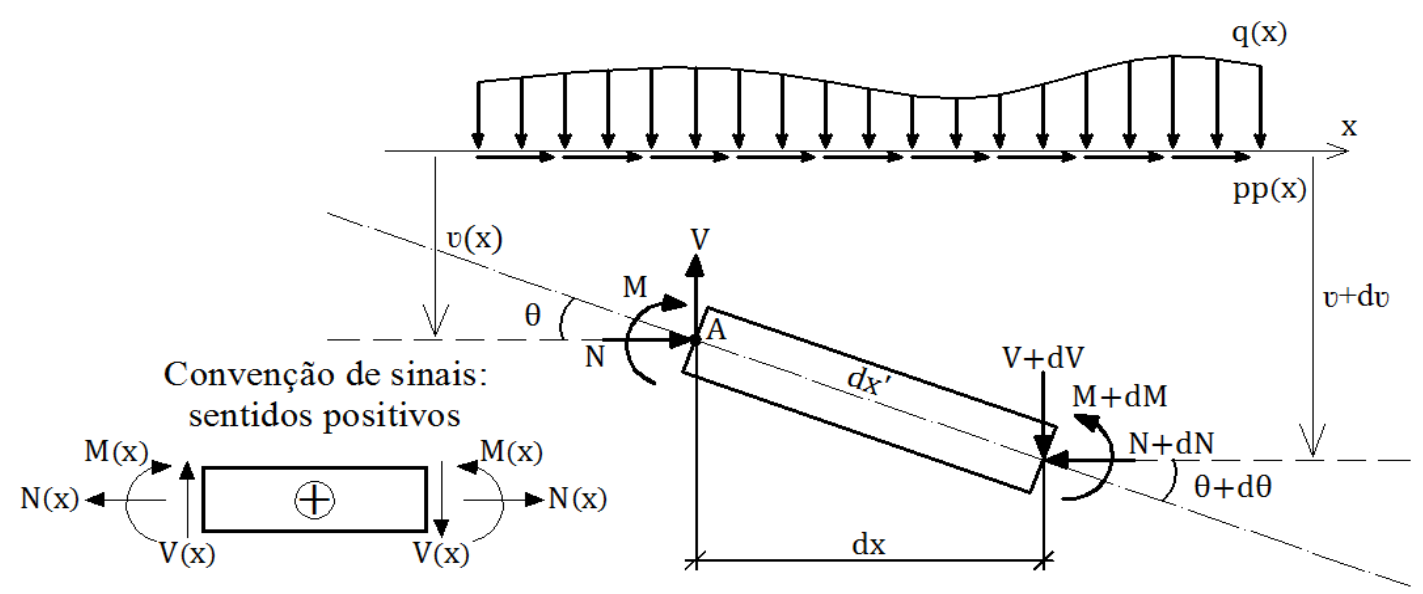

Figura 1 - Configuração da viga-coluna.

Desprezando-se as deformações por cisalhamento e considerando-se a teoria das pequenas deformações, para o trecho de torre, o momento fletor interno $\mathfrak{M}=\mathfrak{M}(\mathrm{x})$ é:

$$
\mathfrak{M}=-E I \frac{d^{2} v}{d x^{2}}
$$

Na Equação (7): E é o módulo de elasticidade longitudinal do material (considerado constante nesta análise) e $I=I(x)$ é a função de momento de inércia da seção transversal da torre. Então, substituindo-se a Equação 7 na Equação 2, onde iguala-se o momento interno ao momento externo, resulta:

$$
-\frac{d}{d x}\left(E \mid \frac{d^{2} v}{d x^{2}}\right)=v+N \frac{d v}{d x}
$$

da qual, derivando-se e substituindo-se a Equação 4 e a Equação 6, tem-se:

$$
\frac{d^{2}}{d x^{2}}\left(E I \frac{d^{2} v}{d x^{2}}\right)-p p \frac{d v}{d x}+N \frac{d^{2} v}{d x^{2}}=q
$$

que é a equação diferencial ordinária da viga-coluna, a qual, uma vez expandida, fica: 


$$
E I \frac{d^{4} v}{d x^{4}}+2 E \frac{d I}{d x} \frac{d^{3} v}{d x^{3}}+\left(E \frac{d^{2} I}{d x^{2}}+N\right) \frac{d^{2} v}{d x^{2}}-p p \frac{d v}{d x}=q
$$

Esta equação diferencial ordinária não homogênea, cuja incógnita é a função de deslocamento transversal da torre com seção transversal variável e que considera a influência da carga axial, permite analisar matematicamente a torre engastada na base (análise não linear geométrica). Entretanto, levando-se em conta que não se tem uma solução analítica, esta expressão é resolvida via método das diferenças finitas.

\subsection{Método energético}

Uma importante questão para o projeto da torre pauta-se no caso homogêneo da Equação 10, a partir do qual se pretende obter a carga de flambagem da torre (análise linear de estabilidade). Um método aproximado para a obtenção da carga de flambagem fundamenta-se no balanço energético, logo, considera-se uma forma modal polinomial (Figura 2) tal que:

$$
v(x)=\sum_{j=0}^{m} A_{j} x^{j}
$$

Na Equação (11): $A_{j}$ são as constantes da forma modal polinomial e utilizando-se das condições de contorno essenciais e natural da base da torre, de forma que:

$$
\begin{gathered}
v(0)=0 ; v^{\prime}(0)=0 \\
v(0)=0 \Rightarrow v^{\prime \prime \prime}(0)=0
\end{gathered}
$$

e, para o topo, as condições de contorno naturais expressas como:

$$
\begin{gathered}
v^{\prime \prime}(L)=0 \\
v(L)=0 \Rightarrow v^{\prime \prime \prime}(L)=-\frac{N(L)}{E I(L)} v^{\prime}(L)=-\frac{P}{E I(L)} v^{\prime}(L)=-\bar{\alpha}^{2} v^{\prime}(L)
\end{gathered}
$$

e, ainda, uma condição de contorno acessória, no topo, definida mediante:

$$
v(L)=\delta
$$

Na Equação (14): $\delta$ representa o deslocamento transversal no topo da torre e $L$ é o comprimento da estrutura. Então, utilizando-se até a quinta potência $(m=5)$, a 1 a forma modal da torre fica:

$$
v(x)=\frac{\delta}{L^{2}\left(\bar{\alpha}^{2} L^{2}-28\right)}\left[\left(\frac{10}{3} \bar{\alpha}^{2} L^{2}-40\right) x^{2}+\frac{5}{L^{2}}\left(4-\bar{\alpha}^{2} L^{2}\right) x^{4}+\frac{8}{3 L^{3}}\left(\bar{\alpha}^{2} L^{2}-3\right) x^{5}\right]
$$

O trabalho, $\mathrm{T}_{\mathrm{pp}}$, realizado pela carga axial distribuída $\mathrm{pp}$ ao longo da função de deslocamento axial $u=u(x)$ da torre é dado, aproximadamente, por: 


$$
T_{p p}=\int_{0}^{L} p p(x)\left\{\int_{0}^{x} \frac{\left[v^{\prime}(x)\right]^{2}}{2}\right\} d x
$$
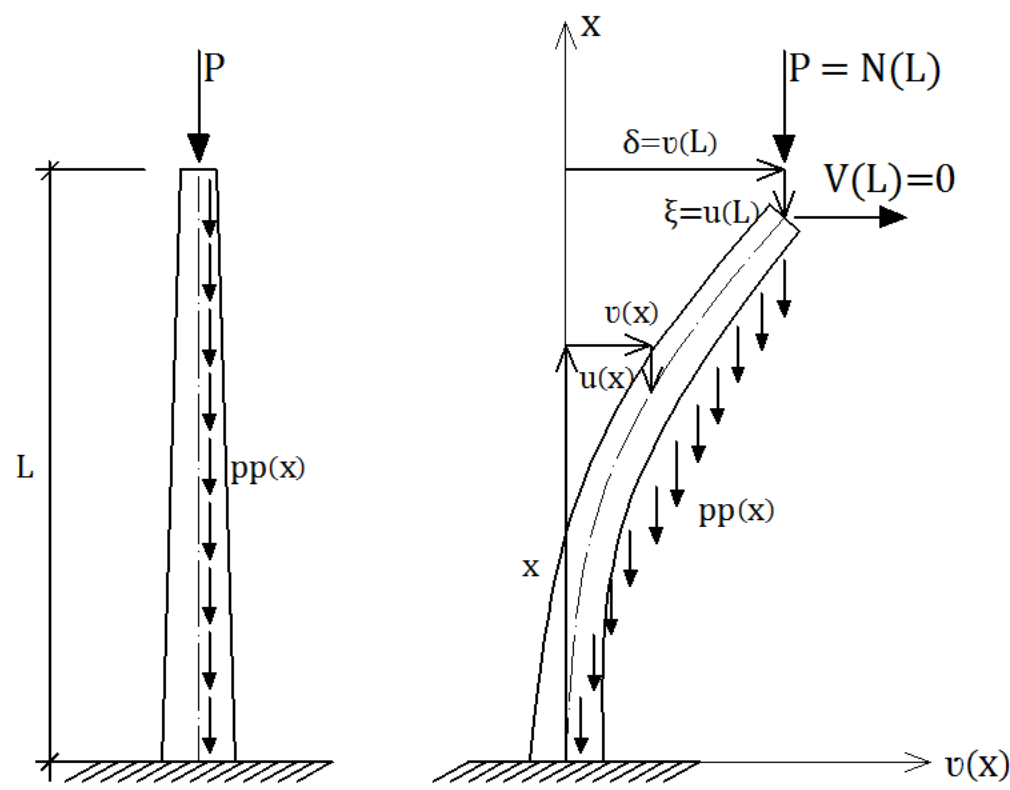

Figura 2 - Forma modal considerada para a torre.

Já o trabalho, $T_{P}$, realizado pela força axial concentrada $P$, aplicada ao topo, ao longo do deslocamento axial $u=u(x)$ da torre é aproximadamente:

$$
T_{P}=\int_{0}^{L} P \frac{\left[v^{\prime}(x)\right]^{2}}{2} d x
$$

De outra parte, a energia de deformação oriunda da flexão, $U$, na qual se desprezam as energias de deformação por cisalhamento e axial, fica:

$$
U=\int_{0}^{L} \frac{E I(x)\left[v^{\prime \prime}(x)\right]^{2}}{2} d x
$$

Para a avaliação das integrais das Equações 16, 17 e 18 são estabelecidos os seguintes vetores contendo as funções relacionadas à variação da seção transversal do tubo da torre ao longo da altura: função de diâmetros $d(x)$, conforme Equação 19; vetor de funções de áreas de seção transversal $A(x)_{i}$, conforme Equação 20; vetor de funções de pesos próprios por unidade de comprimento $p p(x)_{i}$, conforme Equação 21; e vetor de funções de momentos de inércia $\mathrm{I}(\mathrm{x})_{\mathrm{i}}$, conforme Equação 22 . Tais vetores foram estabelecidos a partir do vetor de espessuras da parede do tubo esp $p_{\mathrm{i}}$, que considera o processo de fabricação da torre, no qual são utilizadas chapas grossas com espessuras comerciais calandradas para formar o tubo da torre: 


$$
d(x)=\frac{d_{\text {base }}}{L}\left[L-\left(1-\frac{d_{\text {topo }}}{d_{\text {base }}}\right) x\right]
$$

Na Equação (19): $d_{\text {base }}$ e $d_{\text {topo }}$ são os diâmetros médios do tubo na base e no topo;

$$
A(x)_{i}=\pi d(x) e^{e s p} p_{i}
$$

Na Equação (20): espi é o vetor de espessura da parede do tubo da torre avaliado nos níveis i ao longo do comprimento;

$$
p p(x)_{i}=\frac{p p_{\text {base }}}{L}\left[L-\left(1-\frac{d_{\text {topo }}}{d_{\text {base }}}\right) x\right] \frac{e^{e s p_{i}}}{e_{\text {base }}}
$$

Na Equação (21): pp base é o peso próprio por unidade de comprimento na base da torre e ebase é a espessura da parede do tubo na base da torre;

$$
\mathrm{I}(\mathrm{x})_{\mathrm{i}}=\frac{\pi}{64}\left\{\left[\mathrm{~d}(\mathrm{x})_{\mathrm{i}}+\operatorname{esp}_{\mathrm{i}}\right]^{4}-\left[\mathrm{d}(\mathrm{x})_{\mathrm{i}}-\operatorname{esp}_{\mathrm{i}}\right]^{4}\right\}
$$

Estabelecendo-se uma relação $\beta$ entre o peso próprio por unidade de comprimento na base da torre e a força axial concentrada P aplicada ao topo da torre, tem-se:

$$
\beta=\frac{p p_{\text {base }} L}{P}
$$

aplicando-se os vetores de funções das Equações 19, 20, 21 e 22 na integral da Equação 18, obtém-se a seguinte expressão para energia de deformação por flexão:

$$
U=\frac{E}{2} \sum_{i=0}^{n-1}\left[\int_{i \cdot h}^{(i+1) \cdot h} I(x)_{i}\left[v^{\prime \prime}(x)\right]^{2} d x\right]
$$

Na Equação (24): $\mathrm{n}$ é o número de subdivisões escolhido para a torre e $\mathrm{h}$ é o comprimento do trecho de torre analisado.

Ademais, utilizando-se as Equações 19, 20, 21, 22 e 23 nas Equações 16 e 17, obtém-se a expressão do trabalho realizado pelas cargas:

$$
T=\frac{P}{2}\left\{\frac{\beta}{L^{2}} \sum_{i=0}^{n-1}\left[\int_{i \cdot h}^{(i+1) \cdot h}\left[L-\left(1-\frac{d_{\text {topo }}}{d_{\text {base }}}\right) x\right] \frac{e^{-s p_{i}}}{e_{\text {base }}}\left[\int_{0}^{x}\left[v^{\prime}(x)\right]^{2} d x\right] d x\right]+\int_{0}^{L}\left[v^{\prime}(x)\right]^{2} d x\right\}
$$

Finalmente, mediante o princípio de conservação de energia, $T=U$, escreve-se $o$ quociente de Rayleigh para a carga de flambagem:

$$
P_{C R}=\frac{E \sum_{i=0}^{n-1}\left[\int_{i \cdot h}^{(i+1) \cdot h} I(x)_{i}\left[v^{\prime \prime}(x)\right]^{2} d x\right]}{\frac{\beta}{L^{2}} \sum_{i=0}^{n-1}\left\{\int_{i \cdot h}^{(i+1) \cdot h}\left[L-\left(1-\frac{d_{\text {topo }}}{d_{\text {base }}}\right) x\right] \frac{e^{e s p_{i}}}{e_{\text {base }}}\left[\int_{0}^{x}\left[v^{\prime}(x)\right]^{2} d x\right] d x\right\}+\int_{0}^{L}\left[v^{\prime}(x)\right]^{2} d x}
$$




\section{Projeto do modelo}

O modelo de torre tubular de aço analisado neste trabalho pautou-se no projeto estático da torre considerando-se as prescrições normativas dos seguintes códigos: ABNT NBR 6123:1988; ABNT NBR 8800:2008; ABNT NBR 6118:2014; ABNT NBR IEC 61400-1:2008; EN 1991-1-4:2005; EN 1993-3-2:2006. Então, considerouse uma torre tubular de aço S355J2, segundo as especificações da EN 10025-2:2004, a qual dá suporte a um aerogerador no padrão SWT-3.2-113 (Siemens, 2014), conforme características especificadas na Tabela 1.

Tabela 1 - Dados do padrão do aerogerador selecionado. Fonte: Siemens (2014).

Tipo de parâmetro

\begin{tabular}{cc}
\hline $\begin{array}{c}\text { Classe segundo IEC } \\
\text { (International Electrotechnical Commission) }\end{array}$ & IIA \\
\hline Potência nominal (MW) & 3,2 \\
\hline Diâmetro do rotor $(\mathrm{m})$ & 113,0 \\
\hline Comprimento da pá (m) & 55,0 \\
\hline Área varrida pelo rotor ( $\left.\mathrm{m}^{2}\right)$ & 10000 \\
\hline Altura do cubo do rotor $(\mathrm{m})$ & $79,5-142,0$ (usou-se 122,5 $\mathrm{m})$ \\
\hline Regulação de potência & Ângulo de passo regulado \\
\hline Energia elétrica produzida anualmente a 8,5 m/s & $14402 \mathrm{MWh}$ \\
\hline Peso da nacele (tf) & 78 \\
\hline Peso do rotor (tf) & 67 \\
\hline
\end{tabular}

Extrapolando-se os resultados de forças e momentos transmitidos ao topo da torre (Figura 3), em condições eólicas normais e extremas, estabelecidos por Asibor et al. (2015) que utilizaram o software GL bladed e por Lavassas et al. (2003) que utilizaram dados fornecidos pelo fabricante, obtêm-se os valores de forças e momentos máximos aplicados ao topo da torre, conforme Tabela 2.

Além do carregamento aplicado ao topo da torre, utilizam-se as ações aplicadas ao longo do comprimento da torre, ou seja:

i. Carga permanente da torre distribuída axialmente;

ii. Cargas dos equipamentos dispostos ao longo da altura da torre (equipamentos das instalações elétricas a exemplo de: cabos para transmissão de energia elétrica, transformador, sistema de climatização, sistema de iluminação, sistema de controle; e, equipamentos de segurança para manutenção tais como: sistema de ascensão/escadas, plataformas intermediárias etc.) também dispostas axialmente; 
iii. Ação do vento orientada radialmente (segundo recomendações das ABNT NBR 6123:1988; ABNT NBR IEC 61400-1:2008; EN 1991-1-4:2005) e ao longo da altura da torre (Figura 4): utilizando uma velocidade básica de vento igual a $35 \mathrm{~m} / \mathrm{s}$ (valor máximo de velocidade para o estado de Pernambuco, onde se idealiza a implantação do parque eólico);

iv. Força lateral distribuída ao longo da altura da torre equivalente ao desaprumo de L/2000 compatível ao processo de fabricação e montagem da mesma.

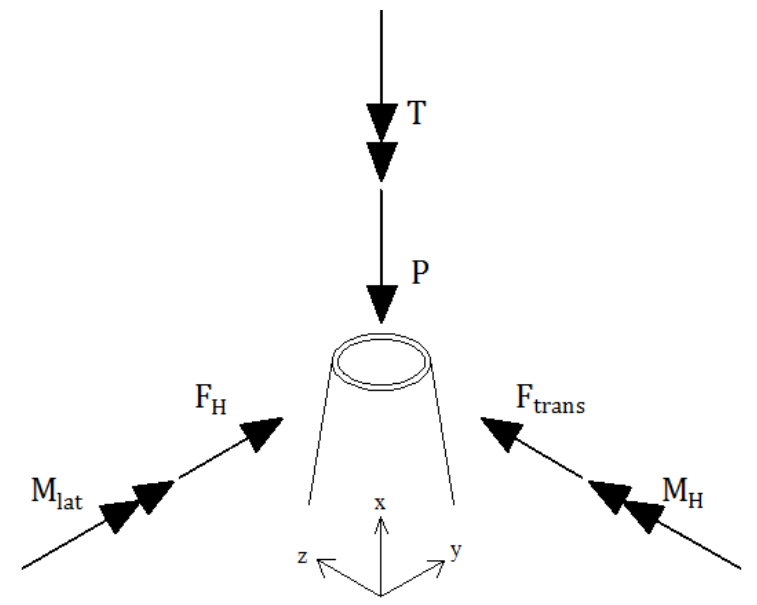

Figura 3 - Representação das forças e momentos aplicados ao topo da torre.

Tabela 2 - Carregamento aplicado ao topo da torre.

\begin{tabular}{cccccc}
\hline $\mathbf{P}(\mathbf{N})$ & $\mathbf{F}_{\mathbf{H}}(\mathbf{N})$ & $\mathbf{F}_{\text {trans }}$ (N) & $\mathbf{M}_{\mathbf{H}}$ (N.m) & $\mathbf{M}_{\text {lat }}$ (N.m) & $\mathbf{T}$ (N.m) \\
\hline 4299033,45 & 662186,43 & 32106,07 & 46644600,79 & 4147943,60 & 1985250,43 \\
\hline
\end{tabular}

A torre tubular projetada tem uma altura total de $120 \mathrm{~m}$, é formada por uma estrutura tronco-cônica com diâmetro na base de 6,5 m e no topo de 3,5 $\mathrm{m}$ e a espessura da parede da torre tubular varia de $2 "$ na base para $11 / 4 "$ no topo (Figura 5).

Para o transporte, o içamento e a montagem, a torre é subdividida em quatro partes de $30 \mathrm{~m}$ que são conectadas por meio de flanges (anéis de conexão entre segmentos da torre) unidos com parafusos pré-tracionados de alta resistência classe ISO 10.9, segundo especificações da ISO 7411:1984, em ligações por atrito. Adicionalmente, utilizam-se nestas ligações porcas, segundo recomendações da ISO 4775:1984, e arruelas, normatizadas pela ISO 7415:1984. Os flanges são posicionados/soldados de maneira que a furação dos mesmos se encontre na parte interior do tubo, permitindo fácil acesso para manutenção dos parafusos. Uma configuração similar é utilizada na ligação entre o flange azimutal da torre e o anel de direcionamento da nacele, neste caso, a especificação do flange de topo é feita de acordo com o fabricante do anel de 
direcionamento da nacele. O flange da base da torre é fixado à fundação (neste caso, uma sapata) por barras de ancoragem (chumbadores) arranjadas concentricamente em ambos os lados da parede da torre tubular.

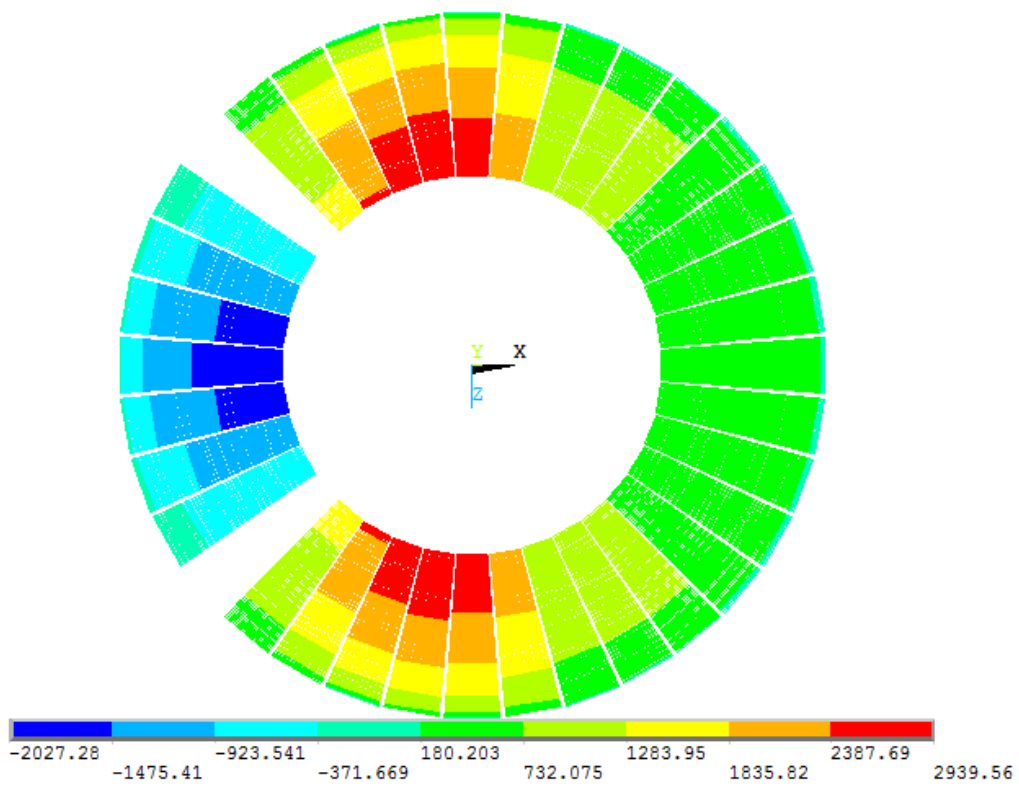

(a) Vista superior da distribuição da pressão de vento $\left(\mathrm{N} / \mathrm{m}^{2}\right)$.

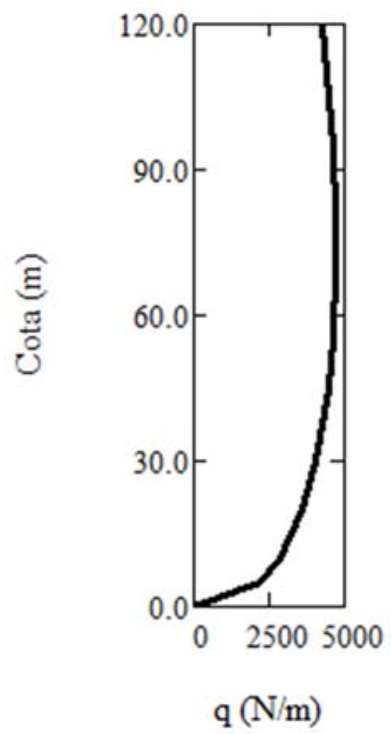

(b) Perfil da ação transversal do vento.

Figura 4 - Distribuição da ação do vento atuante na torre.

A fundação da torre tubular consiste em uma sapata circular, de concreto armado com $\mathrm{f}_{\mathrm{ck}}=30 \mathrm{MPa}$, formada por: um cilindro de $26,0 \mathrm{~m}$ de diâmetro e 0,5 $\mathrm{m}$ altura apoiado sobre solo; acima deste é disposto um segmento com altura de 2,5 m de formato troncocônico no qual o diâmetro varia, ao longo da altura, de $26,0 \mathrm{~m}$ a 7,2 m; e, por fim, temse um pedestal com diâmetro de 7,2 m e altura de 0,75 m (Figura 5). Para a definição das dimensões da sapata foi utilizado DNV/Ris $\varnothing$ (2002), a partir do qual foram analisados os esforços de tombamento e deslizamento da estrutura, como um todo, e as tensões atuantes em comparação com a tensão admissível do solo.

A tensão admissível do solo de assentamento da sapata foi calculada a partir dos métodos teóricos de Meyerhof, Hansen e Vesic' (Bowles, 1996), considerando-se as seguintes propriedades: tipo SW (sand well graded) segundo o Sistema Unificado de Classificação, ângulo de atrito interno de 30 e e peso específico aparente igual a $19 \mathrm{kN} / \mathrm{m}^{3}$. Em seguida, de acordo com a ABNT NBR 6118:2014, foram dimensionadas as armaduras longitudinais superiores e inferiores e a armadura transversal; além disto, 
foram feitas as verificações de punção, abertura de fissuras e ancoragem das barras de reforço.

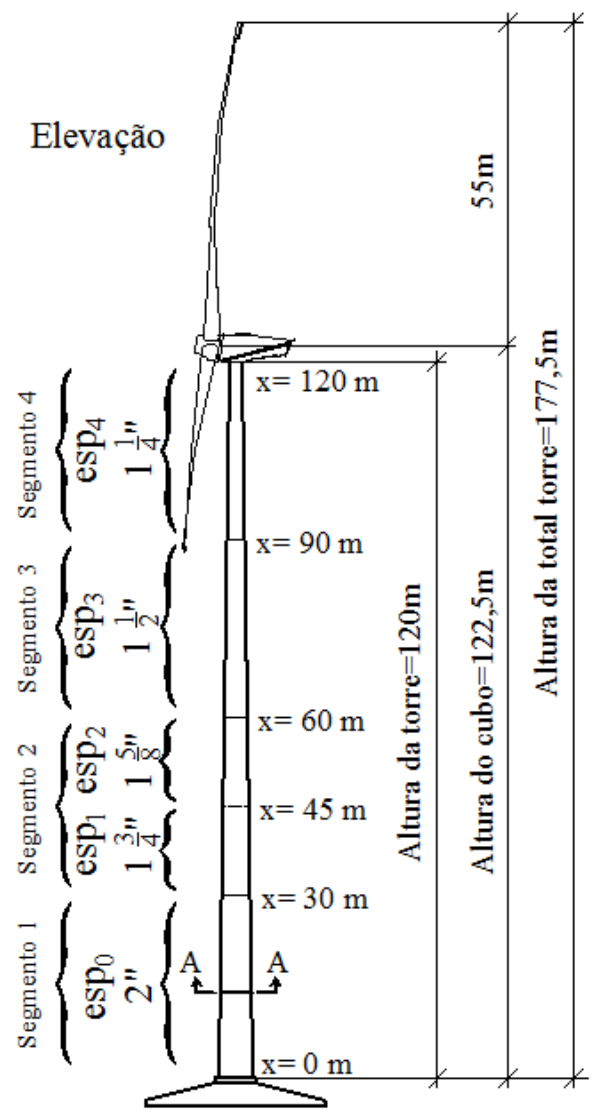

Corte A-A: seção transversal da torre

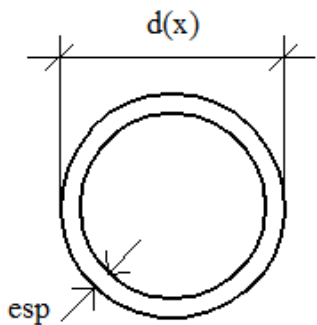

Sapata da torre: corte

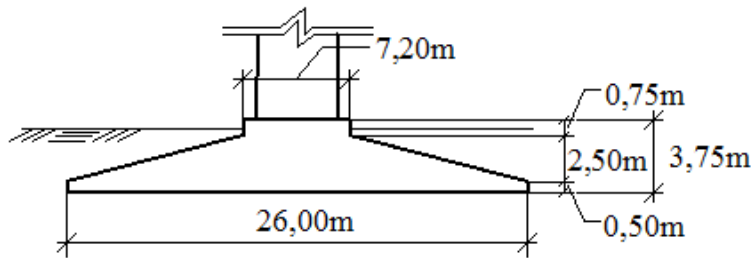

Figura 5 - Esquema do projeto da torre.

\section{Modelagem no software ANSYS}

A análise estrutural e o projeto da torre tubular de aço foram elaborados mediante o método dos elementos finitos (M. E. F.), considerando-se materiais de comportamento elástico, do ponto de vista físico, e não linear, do ponto de vista geométrico.

Inicialmente, foi criado um modelo com elementos de barra com 4 graus de liberdade por nó, no software Mathcad 14, no qual se levam em conta as energias de deformações por flexão e por corte, bem como a influência das cargas axiais na deformação transversal da torre.

Adicionalmente, foi criado um modelo de elementos finitos no software ANSYS (2012) r.14.5, no qual se considerou a torre engastada na base com 7272 elementos de casca, designado por SHELL 181, com 4 nós e com 6 graus de liberdade por nó. O motivo que levou à utilização de um modelo em elementos finitos detalhado e outro em elementos 
de barra, simplificado, portanto, foi a necessidade de avaliar a confiabilidade e a precisão dos resultados numéricos obtidos.

O modelo com elementos finitos de casca foi complementado simulando-se a torre em conjunto com sua fundação. Para tal, a sapata foi modelada com 11766 elementos sólidos tetraédricos, designados por SOLID 186, com 20 nós e 3 graus de liberdade de translação por nó. Além disto, com o objetivo de avaliar a interação solo-estrutura, a reação elástica do solo foi modelada com 2145 elementos de mola com rigidez axial, colocados na base da sapata e designados por COMBIN 14. A rigidez destes elementos foi avaliada a partir do valor médio do coeficiente de reação vertical, de uma areia com densidade relativa média, proposto por Terzaghi (1955). Assim, o valor do coeficiente reação vertical do solo, que é igual a $45023 \mathrm{kN} / \mathrm{m}^{3}$, foi multiplicado pela área de influência de cada nó da base da sapata que está em contato com o terreno.

\section{Resultados e discussões}

Apresentam-se, na Figura 6, as representações gráficas das expressões de interação correspondentes à verificação das seções transversais do modelo de torre projetado, no qual se considera o modelo com elementos finitos de barra para obtenção dos esforços solicitantes de cálculo de 2 a ordem geométrico, sem redução das rigidezes à flexão e axial, uma vez que, a análise realizada é elástica linear (análise física linear). Adicionalmente, observam-se, na Figura 6, os degraus resultantes da mudança brusca de espessura da chapa que forma a torre, nas cotas de 30, 45, 60 e $90 \mathrm{~m}$. Verifica-se, ainda, um aumento dos valores da expressão de interação (Figura 6-b) com a altura da torre, uma vez que se tem uma diminuição dos diâmetros e das espessuras das chapas calandradas em uma proporção maior do que a diminuição dos esforços ao longo da altura. Com relação aos esforços cisalhantes, as seções mais solicitadas apresentaram valores de 4,7\% entre esforços solicitantes e resistentes, não sendo, portanto, determinantes para o dimensionamento.

O máximo valor da expressão de interação é de 74,7\% (Figura 6-b). Ou seja, a seção mais solicitada conta ainda com $25,3 \%$ de capacidade resistente. No entanto, neste estudo não foram avaliados critérios referentes às ações dinâmicas, tais como fadiga nos elementos que compõem a torre (chapas, soldas, parafusos). Adicionalmente, verificou- 
se o estado limite de serviço de deslocamentos máximos no topo da torre, pois é necessário obedecer às limitações de deslocamentos estabelecidas pelos fabricantes dos equipamentos que se encontram na nacele. Além disto, limitam-se os deslocamentos da torre para evitar o contato das pás do aerogerador com a torre de sustentação. Assim, utilizou-se um limite de L/70 para o deslocamento no topo da torre, que, neste estudo, é de $1,70 \mathrm{~m}$.

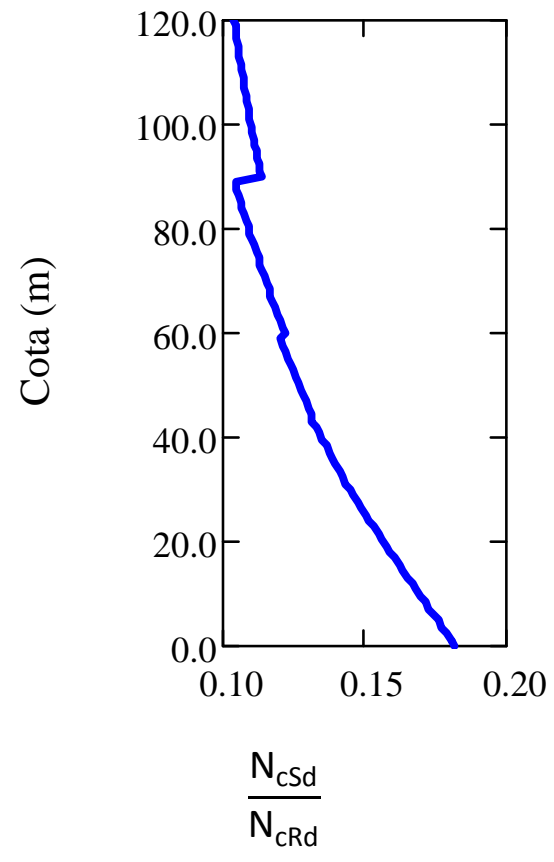

(a) Relação entre os esforços axiais de compressão solicitantes e resistentes.

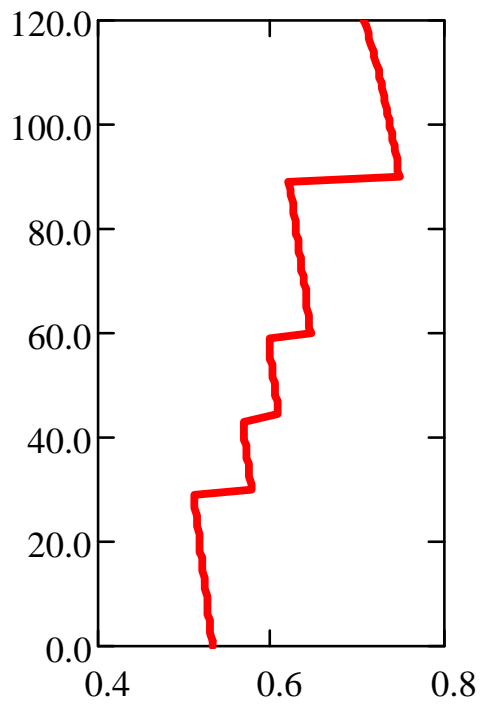

Int

Figura 6 - Representação das expressões de verificação das seções transversais da torre.

Na Figura 6:

$\mathrm{N}_{\mathrm{CRd}}$ é o esforço resistente de cálculo à compressão simples;

$\mathrm{N}_{\mathrm{csd}}$ é o esforço solicitante de cálculo à compressão simples;

Int $=\left\{\begin{array}{l}\frac{N_{c S d}}{N_{c R d}}+\frac{8}{9}\left(\frac{M_{H_{-} S d}}{M_{R d}}+\frac{M_{\text {lat_sd }}}{M_{R d}}\right) \text { para } \frac{N_{c S d}}{N_{c R d}} \geq 0,2 \\ \frac{N_{c S d}}{2 N_{c R d}}+\left(\frac{M_{H_{-} S d}}{M_{R d}}+\frac{M_{\text {lat_sd }}}{M_{\text {Rd }}}\right) \text { para } \frac{N_{c S d}}{N_{c R d}}<0,2\end{array}\right.$

MRd é o momento fletor resistente de cálculo;

$\mathrm{M}_{\mathrm{H}_{-} \mathrm{Sd}}$ é o momento fletor solicitante de cálculo segundo o eixo $z$ da Figura 3;

$M_{\text {lat_sd }}$ é o momento fletor solicitante cálculo segundo o eixo y da Figura 3. 
Para o cálculo do esforço resistente à compressão simples utilizou-se o valor de carga de flambagem (autovalor associado à parcela homogênea da equação diferencial 10 desenvolvida), calculado conforme o método energético descrito no item 2.2, igual a 99607,4 kN para o modelo engastado na base. Adicionalmente, na Figura 7, mostramse os modos de instabilidade do modelo de torre engastado na base e discretizado com elementos finitos de casca. 0 primeiro (Figura 7-a) e o segundo modos são referentes aos primeiros modos de flexão nos planos $\mathrm{YZ}$ e $\mathrm{XY}$, respectivamente (o eixo $\mathrm{Y}$ está posto na vertical no ANSYS, 2012). O terceiro (Figura 7-b) e o quarto modos referem-se a outros dois modos de flexão nos planos $Y Z$ e $X Y$, respectivamente. A partir do quinto modo, caracterizado por três semiondas (Figura 7-c), tem-se uma série de modos de flambagem locais do tubo da torre (com número crescente de semiondas), que não são capturados no modelo com elementos finitos de barra. Estes são modos de flambagem locais acoplados, pois enquanto em um modo de flambagem flexional têm-se deslocamentos segundo um determinado eixo, nos modos de flambagem locais há deslocamentos em mais de um eixo coordenado; entretanto, o quinto modo tem um autovalor correspondente 6,4 vezes maior que o autovalor fundamental (do 10 modo), o que determina a menor importância destes modos superiores (a partir do quinto) à análise de estabilidade linear da torre. Vale salientar que o posicionamento dos modos locais nos modos de vibração da torre está intrinsicamente relacionado com o nível de esbeltez local do tubo que forma a torre, ou seja, com a relação diâmetro/espessura da parede do tubo.

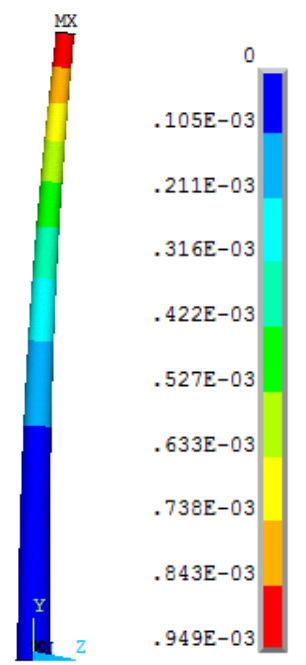

(a) 1 을 modo.

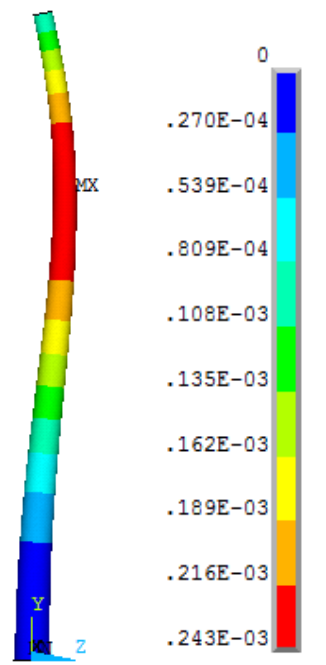

(b) 3 ㅇ modo.

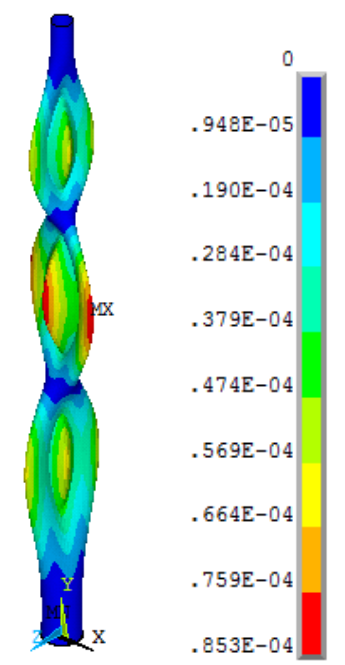

(c) 5o modo.

Figura 7 - Modos de instabilidade da torre. 
Para os modelos engastados na base, elaborados com elementos finitos de barra (em que se considerou a não linearidade geométrica, mediante a matriz de rigidez geométrica) e via mecânica dos meios contínuos, não houve diferença significativa entre os deslocamentos calculados (Tabela 3). O exemplar engastado na base e modelado com elementos finitos de casca, no software ANSYS (2012), apresentou um deslocamento transversal de 1a ordem no topo da torre praticamente igual ao dos dois anteriores; além de um deslocamento de $2^{\mathrm{a}}$ ordem $1 \%$ superior aos outros dois modelos com base engatada. Por fim, o modelo com base flexível apresentou deslocamentos transversais de 1 a ordem e 2 a ordem no topo da torre, pelo menos, 3,6\% e 4,0\% superiores aos deslocamentos dos modelos com base engastada, respectivamente. Na Tabela 3 apresenta-se, também, a relação entre os deslocamentos transversais de 2 a ordem e de 1a ordem do topo da torre (suscetibilidade aos efeitos de 2 a ordem ou grau de deslocabilidade da estrutura). Salienta-se que, apesar de em todos os casos estudados a estrutura ser classificada como de pequena deslocabilidade (valores abaixo de 1,1), o incremento de deslocamentos é significativo à análise de estabilidade e ao aumento dos esforços solicitantes necessários ao projeto da torre.

Tabela 3 - Deslocamentos transversais do topo da torre $(\mathrm{m})$.

\begin{tabular}{|c|c|c|c|c|c|c|c|}
\hline \multicolumn{6}{|c|}{ Base engastada } & \multirow{2}{*}{\multicolumn{2}{|c|}{$\begin{array}{c}\text { Base flexível } \\
\text { E. F. casca-sapata }\end{array}$}} \\
\hline \multicolumn{2}{|c|}{ M.M.C } & \multicolumn{2}{|c|}{ E. F. barra } & \multicolumn{2}{|c|}{ E. F. casca } & & \\
\hline 1a ord. & 2a ord. & 1a ord. & 2a ord. & 1a ord. & 2a ord. & 1a ord. & 2a ord. \\
\hline 1,50669 & 1,59969 & 1,50869 & 1,59930 & 1,50647 & 1,61563 & 1,56309 & 1,68092 \\
\hline \multicolumn{8}{|c|}{ Graus de deslocabilidade } \\
\hline \multicolumn{2}{|c|}{1,06172} & \multicolumn{2}{|c|}{1,06006} & \multicolumn{2}{|c|}{1,07246} & \multicolumn{2}{|c|}{1,07538} \\
\hline
\end{tabular}

Para a análise da estabilidade não linear geométrica transversal da torre tubular de aço, em conjunto com a sapata e considerando-se a interação solo-estrutura, foram verificados, numa primeira etapa da análise, se os elementos finitos de mola que ligam a sapata ao terreno se encontravam tracionados ou comprimidos sob a aplicação das ações descritas no item 3. Em seguida, iterativamente, nas etapas subsequentes, as molas tracionadas foram sendo desativadas até alcançar o equilíbrio da estrutura apoiada sobre o terreno deformável (Figura 8). Observa-se que 538 dos 2145 nós da base da sapata têm as molas desativadas, desta forma, $25 \%$ da área da base da sapata se levanta e fica sem contato com o solo. 


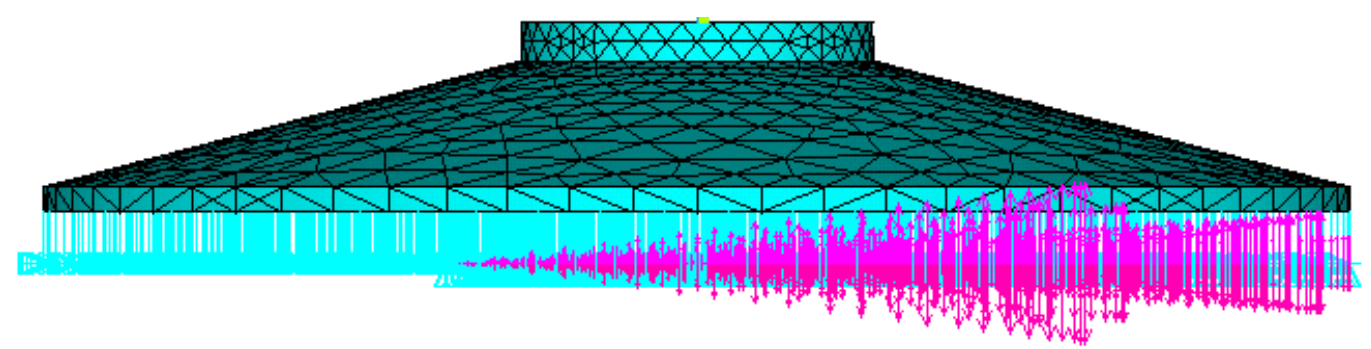

Figura 8 - Representação das reações do solo sobre a sapata.

Na Figura 9 expõe-se uma representação dos deslocamentos verticais da sapata, onde se constatam as molas que não estão trabalhando (em vermelho, laranja e amarelo), ou seja, a região onde a sapata se levanta acima do terreno. Observe-se que o deslocamento vertical máximo para baixo, na borda da sapata, é 3,284 mm. Em virtude da flexibilidade do sistema fundação-solo, que resulta em uma rotação de 0,0289 da base da torre, o deslocamento transversal do topo da torre é aumentado em 6,529 cm (Tabela 3) quando comparado com o modelo de elemento finito de casca engastado na base. Salienta-se que as deformações obtidas (deslocamentos e rotações) poderiam ser ainda maiores no caso desta fundação estar assente em um solo de menor qualidade, uma vez que, o tipo de solo utilizado neste estudo apresenta excelentes propriedades físicas e mecânicas, compatíveis com a região do agreste de Pernambuco, onde se idealiza a implantação do parque eólico.

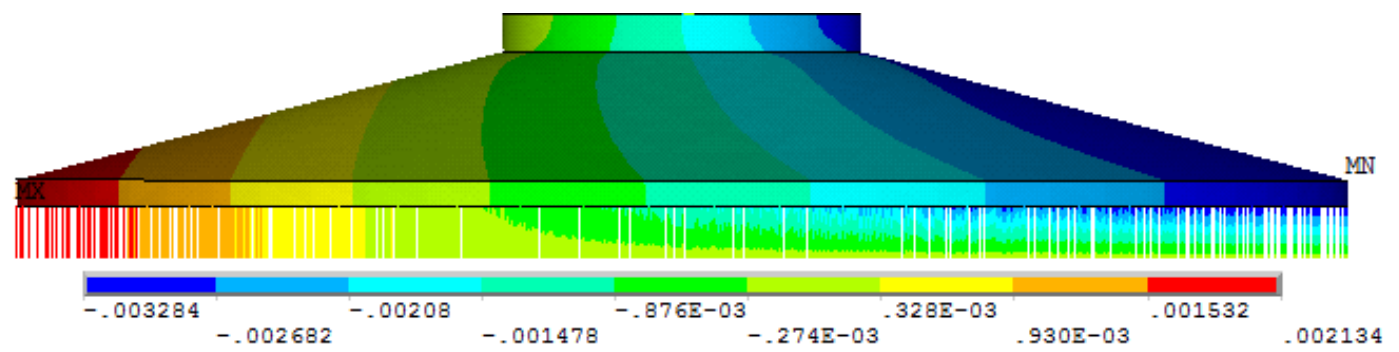

Figura 9 - Deslocamentos verticais da sapata $(\mathrm{m})$.

Na Figura 10 tem-se a distribuição de von Mises para o modelo de torre tubular de aço, no qual considera-se a interação solo-estrutura e a não linearidade geométrica. A máxima tensão de von Mises obtida (182,22 MPa), encontra-se na junção entre os dois últimos segmentos da torre na cota de $90 \mathrm{~m}$, porém, com valor abaixo da tensão admissível (208,82 MPa) do aço utilizado (S355J2); fato que justifica a utilização do modelo linear para a equação constitutiva do aço. Observe-se que o critério determinante para o projeto da torre foi a limitação dos deslocamentos máximos transversais no topo desta. Desta forma, não houve necessidade de empregar modelos de falha, pois nenhum ponto da torre atingiu a tensão de admissível do aço. 


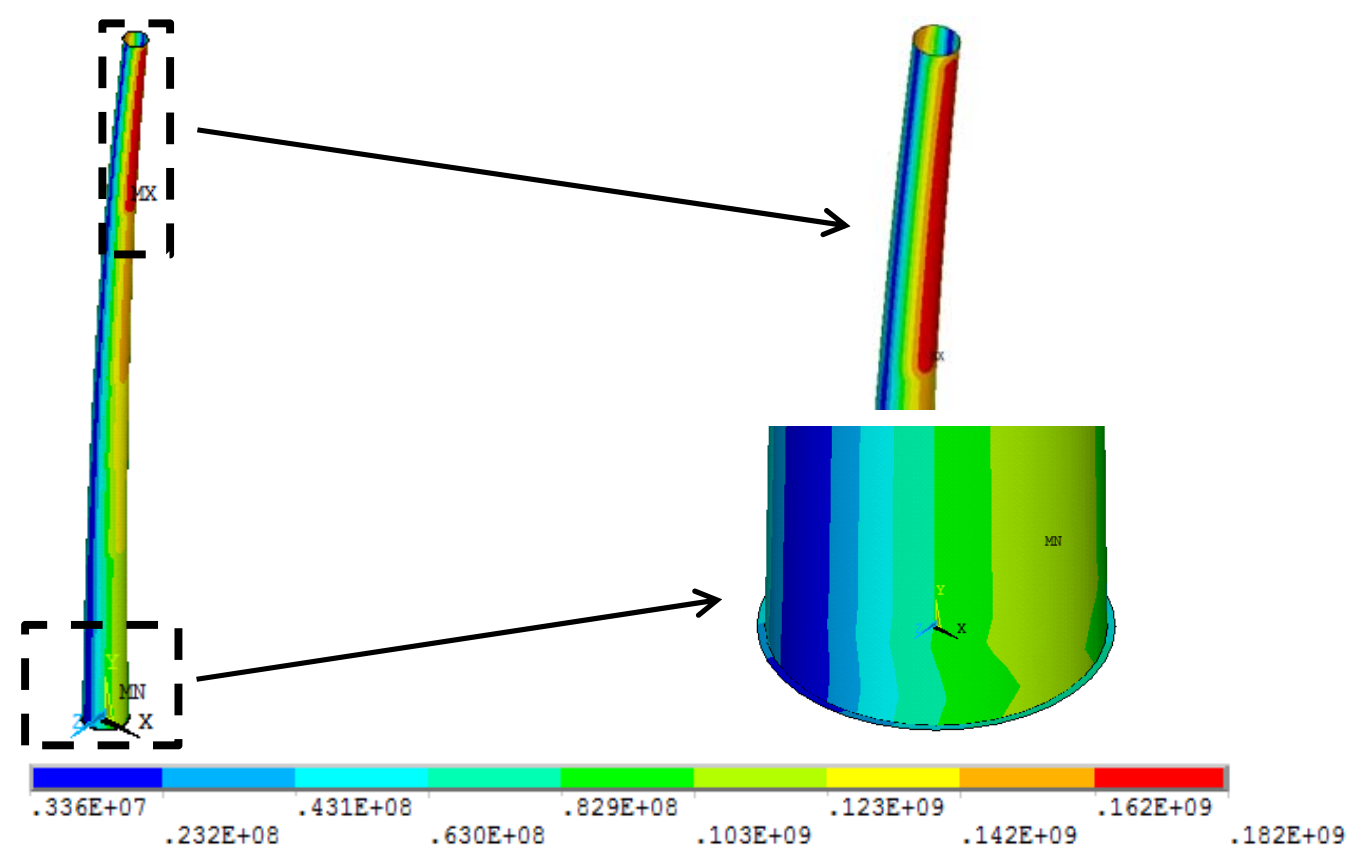

Figura 10 - Distribuição de tensões de von Mises na torre ( $\mathrm{Pa})$.

Por fim, tem-se o detalhamento das ligações parafusadas da torre, nas quais foram utilizados parafusos M36 (ISO 10.9):

i. Ligação da sapata com o flange basal: barras de ancoragem, com 2x144 parafusos (Figura 11-a);

ii. Ligação do flange intermediário 1 com o flange intermediário 2: cota de $30 \mathrm{~m}$ com 180 parafusos (Figura 11-b);

iii. Ligação do flange intermediário 2 com o flange intermediário 3: cota de $60 \mathrm{~m}$ com 144 parafusos (Figura 11-b);

iv. Ligação do flange intermediário 3 com o flange intermediário 4: cota de $90 \mathrm{~m}$ com 144 parafusos (Figura 11-b); e,

v. Ligação do flange azimutal da torre com a cremalheira da nacele: cota de $120 \mathrm{~m}$ com 108 parafusos (Figura 11-c).

Os parafusos de alta resistência utilizados nas ligações entre os flanges foram dimensionados considerando-se ligações por atrito resistentes aos esforços cisalhantes e axiais a serem transmitidos entre os segmentos da torre. Em particular, na base da torre, para a transmissão da força de tração entre a torre de aço e a sapata de concreto armado, calcularam-se a largura e a espessura do anel de aço embutido na base da sapata, no qual são fixadas as barras de ancoragem. A transmissão da força de 
compressão se deu pelo contato entre o flange basal da torre e o anel de aço colocado no topo da sapata, de forma que a torre fica apoiada nas barras de ancoragem que estão contidas lateralmente pelo volume de concreto armado da sapata. No dimensionamento dos anéis da base e do topo da sapata considera-se que a aderência entre as barras de ancoragem (lisas) e o concreto armado da sapata seja nula, assim, a ancoragem é garantida pelo contato dos anéis com o concreto e pela capacidade resistente à flexão destes.

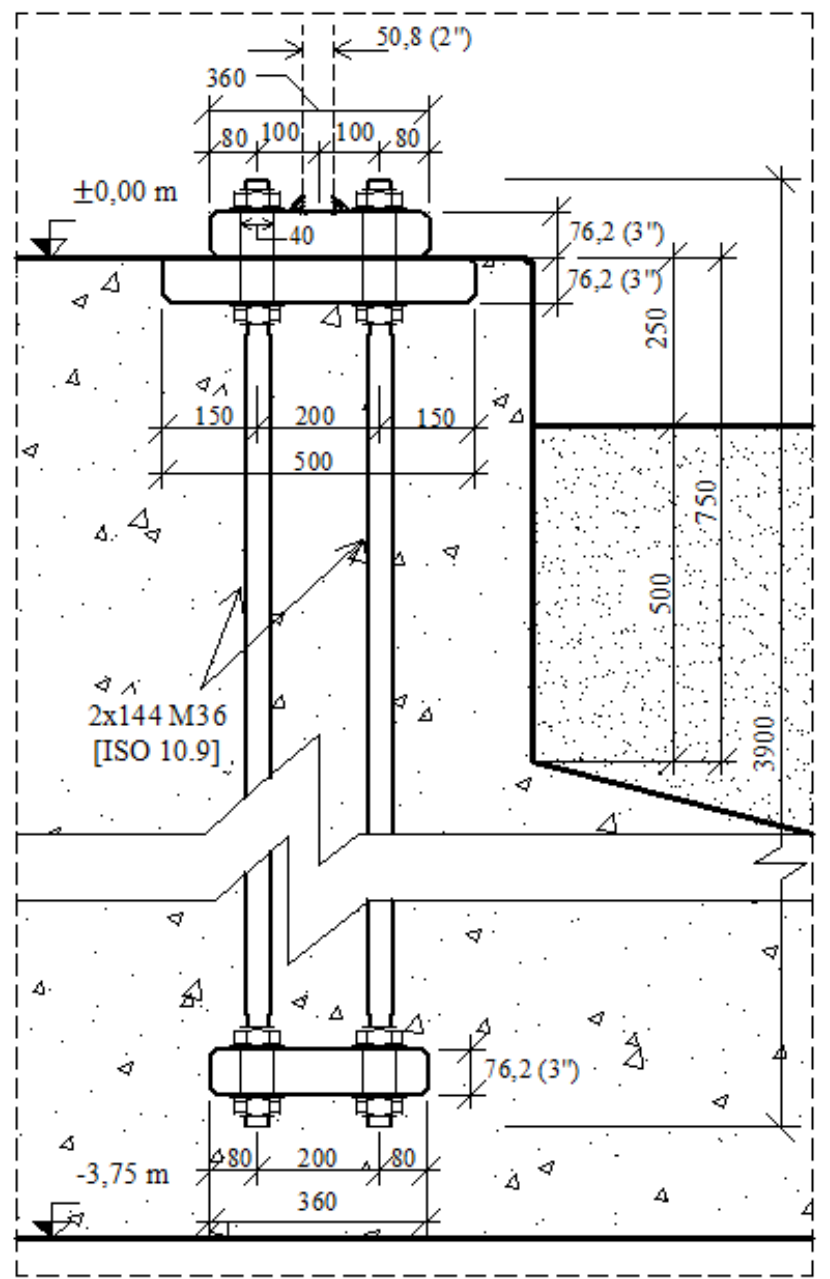

(a) Ligação: sapata - torre (dimensões em mm).

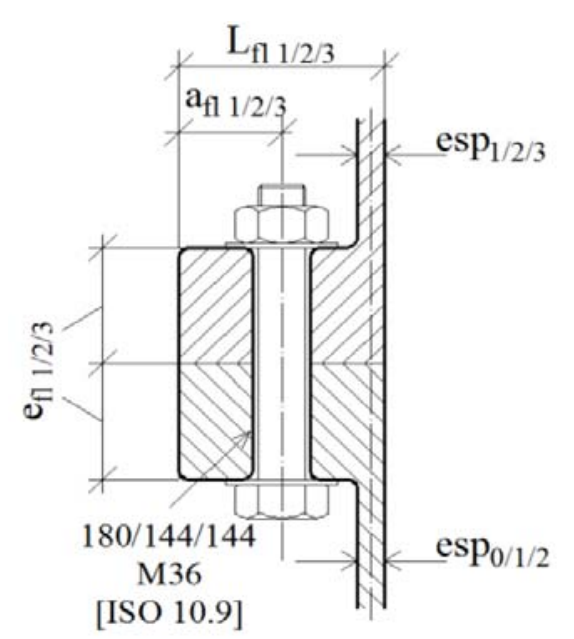

(b) Ligação: flanges intermediários.

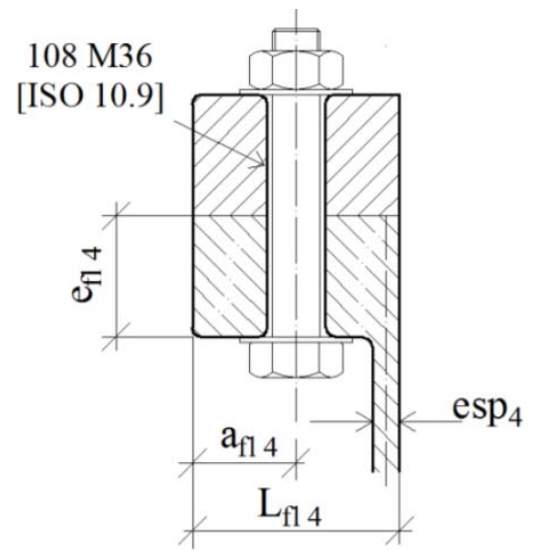

(c) Ligação: flange topo - anel nacele.

Figura 11 - Detalhamento das ligações.

Na Figura 11:

$\operatorname{esp}_{0}\left(2^{\prime \prime}\right), \operatorname{esp}_{1}(13 / 4 "), \operatorname{esp}_{2}(15 / 8 "), \operatorname{esp}_{3}(11 / 2 ")$ e esp 4 (1 1/4 ") são as espessuras da parede do tubo da torre; $e_{f \mid}\left(4^{\prime \prime}\right), e_{f \mid} 2\left(4^{\prime \prime}\right), e_{f \mid} 3\left(31 / 2\right.$ ") e $e_{f \mid} 4$ (4") são as espessuras dos flanges intermediários 1, 2 e 3 e do flange azimutal da torre, respectivamente; Lfl 1 $(28 \mathrm{~cm}), \mathrm{Lfl}_{2}(28 \mathrm{~cm}), \mathrm{Lfl}_{3}(24 \mathrm{~cm})$ e $\mathrm{Lfl}_{4}(24 \mathrm{~cm})$ são as larguras dos flanges intermediários 1, 2 e 3 e do flange azimutal da torre, respectivamente; afl $1(12 \mathrm{~cm})$, afl 2 
$(12 \mathrm{~cm}), a_{f \mid}(11 \mathrm{~cm})$ e $a_{f \mid} 4(11 \mathrm{~cm})$ são as distâncias do eixo do parafuso a borda do flange intermediário 1,2 e 3 e do flange azimutal da torre, respectivamente.

\section{Considerações finais}

A técnica de meio contínuo aplicada à torre tubular de aço do aerogerador mostrou-se adequada para previsão da carga de flambagem (solução da parcela homogênea da equação diferencial), dos esforços e das deformações utilizados para realizar o projeto da torre com seção transversal variável e engastada na base.

O modelo estrutural via elementos finitos foi comparado com o modelo de meio contínuo, resolvido mediante diferenças finitas, observando-se que os resultados obtidos apresentam-se consistentes e muito próximos, o que garante a validade das técnicas numéricas implementadas.

Constatou-se, para o modelo simulado no software ANSYS (2012), em que se considera a interação solo-estrutura, que há estabilidade para o conjunto estrutural torre-sapatasolo, ou seja, o sistema tende para uma deformada final, estável, compatível com o limite de deformações requerido. Adicionalmente, na análise da distribuição de tensões de von Mises, ao longo da torre, não houve necessidade de empregar modelos de falha, pois nenhum ponto da estrutura atingiu a tensão de admissível do aço empregado. Por fim, estes resultados serviram de base para o projeto das ligações entre os segmentos da torre e entre sua base e a sapata.

Os resultados desta pesquisa envolvem contribuições de interesse prático imediato, uma vez que se pretende desenvolver subsídios para análises de estabilidade de torres e fundações para aerogeradores a serem implantados no território brasileiro.

\section{Referências}

ASIBOR, A. I. et al. Wind turbine performance and loading calculations using aero elastic modelling. In: International Congress of Mechanical Engineering, 23, 2015, Rio de Janeiro. Anais... Rio de Janeiro: ABCM, 2015.

ASSOCIAÇÃO BRASILEIRA DE NORMAS TÉCNICAS. NBR 6118: Projeto de estruturas de concreto: Procedimento. Rio de Janeiro, 2014.

ASSOCIAÇÃO BRASILEIRA DE NORMAS TÉCNICAS. NBR 6123: Forças devidas ao vento em edificações. Rio de Janeiro, 1988.

ASSOCIAÇÃO BRASILEIRA DE NORMAS TÉCNICAS. NBR 8800: Projetos de estruturas de aço e de estruturas mistas de aço e concreto de edifícios. Rio de Janeiro, 2008. 
ASSOCIAÇÃO BRASILEIRA DE NORMAS TÉCNICAS. NBR IEC 61400-1: Aerogeradores Parte 1: Requisitos de Projeto. Rio de Janeiro, 2008.

BARIFOUSE, Rafael; SCHREIBER, Mariana. Como o Nordeste virou principal polo da energia eólica no Brasil. BBC BRASIL, São Paulo e Brasília, 13 nov. 2015. Disponível em: $<$ http://www.bbc.com/portuguese/noticias/2015/11/151110_energia_eolica_nordeste_rb>. Acesso em: 27 set. 2017.

BAZEOS, N. et al. Static, seismic and stability analyses of a prototype wind turbine steel tower. Engineering Structures, v. 24, n. 8, p. 1015-1025, 2002.

BOWLES, J. E. Foundation Analysis and Design. 5th ed. Singapore: McGraw-Hill Book Co., 1996. DNV/Ris $\varnothing$. Guidelines for Design of Wind Turbines. 2 ed. Denmark: Jydsk Centraltrykkeri, 2002. ENGSTRÖM, S. et al. Tall Towers For Large Wind Turbines. Estocolmo: Elforsk, 2010.

EUROPEAN COMMITTEE FOR STANDARDIZATION. EN 1991-1-4: Eurocode 1: Actions on structures - Part 1-4: General actions - Wind actions. Brussels, 2005.

EUROPEAN COMMITTEE FOR STANDARDIZATION. EN 1993-3-2: Eurocode 3: Design of steel structures - Part 3-2: Towers, masts and chimneys - Chimneys. Brussels, 2006.

EUROPEAN COMMITTEE FOR STANDARDIZATION. EN 10025-2: Hot rolled products of structural steels - Part 2: Technical delivery conditions for non-alloy structural steels. Brussels, 2004.

HAU, E. Wind Turbines: Fundamentals, Technologies, Application, Economics. 2 ed. Munich (Germany): Springer, 2006.

INTERNATIONAL ORGANIZATION FOR STANDARDIZATION. ISO 7411: Hexagon bolts for highstrength structural bolting with large width across flats (thread lengths according to ISO 888) Product grade C - Property classes 8.8 and 10.9. 1984.

INTERNATIONAL ORGANIZATION FOR STANDARDIZATION. ISO 4775: Hexagon nuts for highstrength structural bolting with large width across flats - Product grade B - Property classes 8 and 10. 1984.

INTERNATIONAL ORGANIZATION FOR STANDARDIZATION. ISO 7415: Plain washers for highstrength structural bolting hardened and tempered. 1984.

LAVASSAS, I. et al. Analysis and design of the prototype of a steel 1-MW Wind turbine tower. Engineering Structures, v. 25, p. 1097-1106, 2003.

SIRQUEIRA, A. S. Comportamento estrutural de torres de aço para suporte de turbinas eólicas. 2008. 112f. Dissertação (Mestrado em Engenharia Civil) - Faculdade de Tecnologia e Ciências, Universidade do Estado do Rio de Janeiro, Rio de Janeiro, 2007.

SWANSON ANALYSIS SYSTEMS Inc. ANSYS Mechanical User's Guide. Release 14.5. Canonsburg (Pennsylvania, USA): South pointe, 275 Technology Drive, PA 15317, 2012.

SIEMENS. Siemens D3 platform - 3.0-MW and 3.2 - MW direct drive wind turbines: Reduced complexity, increased profitability. Erlangen, Germany, 2014. Disponível em: <https://www.energy.siemens.com/br/pool/hq/power-generation/renewables/windpower/platform20brochures/D3\%200nshore\%20brochure_ENGLISH_Apr2014_WEB.pdf>. Acesso em: 12 ago. 2015.

TERZAGHI, K. Evaluation of coefficient of subgrade reaction. Geotechnique, v. 5, n. 4, p. 297326, 1955. 